\title{
Light amplification in dye doped polymer films
}

\author{
Ritty J Nedumpara ${ }^{1}$, K Geetha ${ }^{1,2}$, V J Dann ${ }^{1,2}$, C P G Vallabhan ${ }^{2}$, \\ V P N Nampoori ${ }^{1}$ and P Radhakrishnan ${ }^{1}$ \\ ${ }^{1}$ International School of Photonics, Cochin University of Science and Technology, Cochin \\ 682022 , India \\ ${ }^{2}$ Centre of Excellence in Lasers and Optoelectronic Sciences, Cochin University of Science \\ and Technology, Cochin 682 022, India
}

E-mail: rittynedumpara@yahoo.com

Received 26 August 2006, accepted for publication 20 December 2006

Published 9 January 2007

Online at stacks.iop.org/JOptA/9/174

\begin{abstract}
We report unusual spectral narrowing and laser emission from polymer thin films doped with Coumarin 540 dye. The laser emission from the polymer films is found to be highly dependent upon the excitation length of the medium. Even a short length of $1.75 \mathrm{~mm}$ of the dye doped film gave rise to laser emission with FWHM of $0.3 \mathrm{~nm}$ for a pump intensity of $825 \mathrm{~kW} \mathrm{~cm}^{-2}$. The partial reflections from the broad lateral surfaces of the free standing films provided the optical feedback for the laser emission. Occurrence of well-resolved equally spaced resonant modes confirmed the effect of a Fabry-Perot-like optical cavity between the film surfaces.
\end{abstract}

Keywords: dye doped polymer films, spectral narrowing, resonant modes

\section{Introduction}

The development of solid state dye lasers has paved the way for intense research in incorporating dye molecules into solid matrices with different host materials to get a gain medium of high optical quality and photostability. Earlier investigations were done with inorganic glasses and polymers [1-4]. The latter class of material is found to be very attractive both from the technical and economical point of view with its high optical quality, better chemical compatibility with organic dyes and inexpensive processing techniques. Dye doped polymers, with their wide spectral range of operation, are promising materials for polymer based photonics [5-8]. The tunability and high efficiency of laser dyes make them excellent choice for devices based on dye doped polymer waveguides [7-9]. Development of high gain materials for solid state lasers is still an area of intense research $[10,11]$.

Light amplification and spectral narrowing are studied in various systems both in the liquid and solid forms, where the spectral linewidth is reduced to a value less than $10 \mathrm{~nm}$ [12-18]. Spectral narrowing in most of the systems is explained in terms of amplified spontaneous emission (ASE), where the spontaneously emitted light is amplified by the gain medium as it propagates along the path of the maximum optical gain. Reflections from the internal surfaces can increase the path length or allow multiple passes inside the gain medium which in turn will build up the ASE at a faster rate [19]. Supernarrowing of the laser spectrum is reported for polymer waveguides containing dye cored dendrimers. The mirrorless lasing is attributed to the moderate scattering from the aggregated dendrimers in the polymer matrix [20]. Recently, laser emission studies are reported in polymer microcavities and polymer microring lasers [21, 22]. Investigations on distributed feedback lasing in dye doped polymer thin films are also going on [23].

In this paper we report the unusual spectral narrowing of the emission spectra observed in solid state polymeric films doped with Coumarin 540 dye which has emission in the green region. Here the excitation beam cross section at the film surface is in the form of a stripe. At low pump intensities and at small excitation stripe lengths of the dye doped thin films, the emission spectra exhibited the features of amplified spontaneous emission. When the excitation length was increased, keeping the pump intensity a constant, laser emission was observed with a sudden narrowing in spectral linewidth. The excitation length of the gain medium at which a shift from ASE to laser emission was observed varied with the pump intensity. With a pump intensity of 
Light amplification in dye doped polymer films

$825 \mathrm{~kW} \mathrm{~cm}^{-2}$, laser emission having a linewidth of $0.3 \mathrm{~nm}$ was observed from a $1.75 \mathrm{~mm}$ length of the dye doped thin film. Multiple reflections occurring at the fine surfaces of the thin film produced the effect of a Fabry-Perot optical cavity and provided the feedback necessary for laser emission [24]. We could also observe equally spaced, well-resolved laser resonant modes which confirm the existence of an optical cavity formed by the broad surfaces of the film.

\section{Experimental details}

Solid state thin films were prepared by incorporating Coumarin 540 dye with polymethyl methacrylate (PMMA). Commercially available PMMA of $10 \%$ by weight was dissolved in methyl ethyl ketone. The weight percentage was chosen to get optimum viscosity for the formation of good quality films. The dye was dissolved in this solution at a concentration of $5 \times 10^{-4} \mathrm{M}$. The films were formed on micro glass slides by the free cast evaporation method. When the solvent was fully evaporated, good optical quality films could be peeled off the glass slide. Films of different thickness in the range 80-200 $\mu \mathrm{m}$ were made. The thickness of the films was measured by a micrometer with a least count of $0.001 \mathrm{~mm}$ (Mitutoya No. 193-111 with a digit display). Free-standing films were used for the experiments and the emission spectra were recorded by exciting the sample with $476 \mathrm{~nm}$ radiation from a Quanta Ray MOPO (MOPO 700) pumped by a $Q$ switched Nd:YAG laser at $355 \mathrm{~nm}$ that emits pulses of $7 \mathrm{~ns}$ duration at a repetition rate of $10 \mathrm{~Hz}$. With a cylindrical lens, the pump beam was focused on the sample to form a stripe of dimensions $0.5 \mathrm{~mm} \times 9 \mathrm{~mm}$. A slit was incorporated in the path of the beam between the cylindrical lens and the sample so as to vary the stripe length of the sample. The output was collected from the edge of the film using an optical fibre in a direction normal to the pump beam. The emission spectra were recorded with an Acton monochromator attached with a CCD camera. The emitted beam from the edges of the film was strong and highly directional so that we could collect it without any focusing.

\section{Results and discussions}

\subsection{Amplified spontaneous emission}

The absorption spectra recorded using UV-vis spectrophotometer (JascoV-570) of the dye doped thin film and the dye solution in methyl ethyl ketone is given in figure 1 which shows the characteristic absorption of the dye with a peak at $460 \mathrm{~nm}$. The dependence of the emission intensity and spectral linewidth of the emission, on pump intensity and the excitation length of the pump beam was studied in detail. The spectra were recorded for different pump intensities, varying the excitation length of the medium. Figure 2 shows the emission spectra corresponding to three different pump intensities. The emission spectrum narrowed very gradually with the excitation length at lower pump intensities. For a pump intensity of $47 \mathrm{~kW} \mathrm{~cm}^{-2}$ no significant line narrowing was observed with an excitation length of $6 \mathrm{~mm}$ but for a pump intensity of $158 \mathrm{~kW} \mathrm{~cm}{ }^{-2}$, the FWHM of the emission spectrum was $7 \mathrm{~nm}$ for an excitation length of $2.4 \mathrm{~mm}$. At higher pump intensities, the spectral narrowing occurred more rapidly with a high

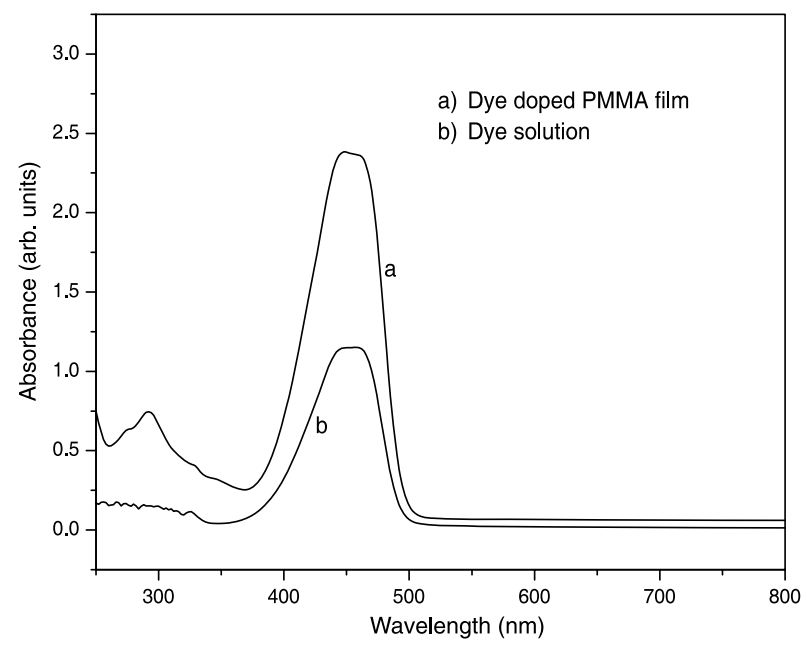

Figure 1. Absorption spectra of dye doped PMMA film and dye solution in methyl ethyl ketone.

gain. While increasing the pump intensity to $825 \mathrm{~kW} \mathrm{~cm}^{-2}$, the FWHM was reduced to $3.5 \mathrm{~nm}$ at an excitation length of $1.5 \mathrm{~mm}$.

When the optically dense dye doped thin film is excited such that it forms a cylindrically shaped active gain medium, the fluorescence emitted by the atoms at one end is strongly amplified by the active medium and it preferentially emits this optical power along the direction of excitation, giving a highly directional output beam [12]. As is evident from figure 2(C), at higher pump intensities the gain increased so that ASE becomes prominent with a reduced linewidth. At lower pump intensities, the emission peaks were observed with the characteristic fluorescence maximum with peaks around $530 \mathrm{~nm}$. But they were red-shifted at higher pump intensities since the self-absorption from the blue side of the spectrum was more prominent at these intensities. With increase in pump intensity a gradual increase in red shift was seen and it was as high as $15 \mathrm{~nm}$ for a pump intensity of $793 \mathrm{~kW} \mathrm{~cm}^{-2}$ (figure 2).

The output emission intensity from the dye doped films was found to be exponentially growing according to the relation for ASE output

$$
I(\lambda)=\frac{A(\lambda) I_{\mathrm{p}}(\exp (g(\lambda) l)-1)}{g(\lambda)}
$$

where $A$ is the Einstein coefficient related to the cross section for spontaneous emission, $I_{\mathrm{p}}$ is the pump intensity, $g$ is the gain coefficient and $l$ is the excitation length of the pump beam [12]. Figure 3 shows the output intensity at the peak of the emission spectrum as a function of excitation length of the gain medium for different pump intensities. We have considered the emission intensity up to an excitation length of $2 \mathrm{~mm}$. The gain coefficient of the dye doped films was calculated by fitting the function for emission intensity of ASE with the experimental data. At low pump intensity, the gain coefficient was less $\left(11 \mathrm{~cm}^{-1}\right)$ but at higher intensities a gain coefficient of $29 \mathrm{~cm}^{-1}$ was observed, which is comparatively large in the case of dye doped films [13]. To understand the nature of ASE in detail, the dependence of emission intensity on incident pump intensity was studied (figure 4). As is evident 


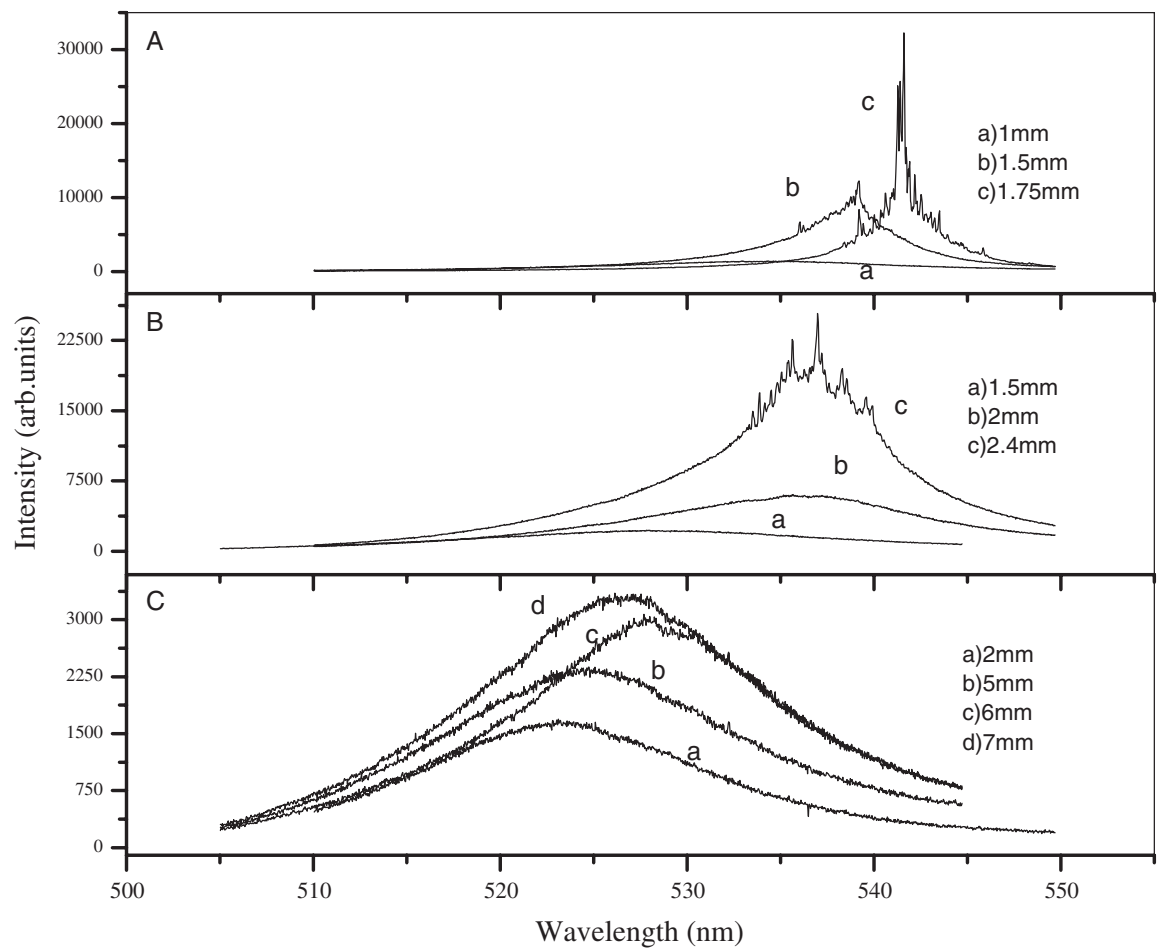

Figure 2. ASE from dye doped films for different excitation lengths of the pump beam at three different power intensities: (A) $32 \mathrm{~kW} \mathrm{~cm}^{-2}$; (B) $158 \mathrm{~kW} \mathrm{~cm}{ }^{-2}$ and (C) $793 \mathrm{~kW} \mathrm{~cm}$. $^{-2}$.

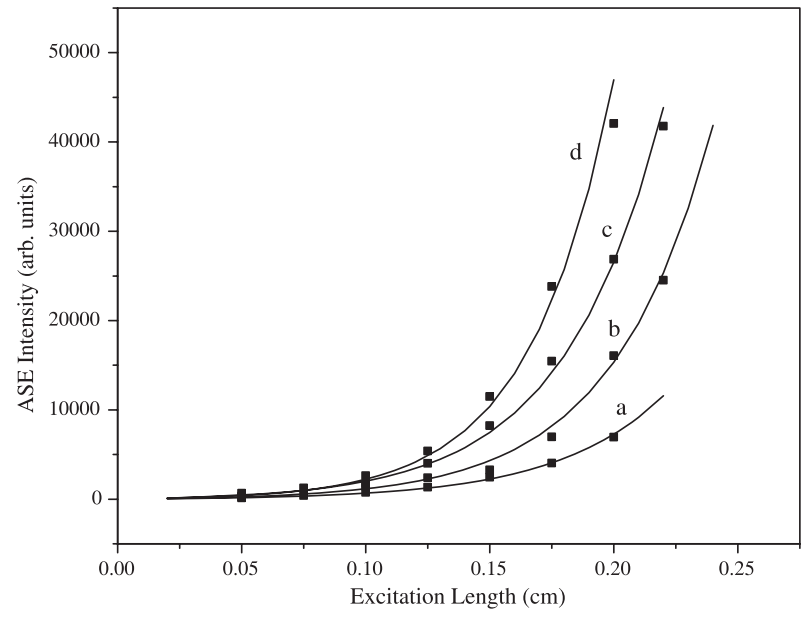

Figure 3. Dependence of emission intensity of ASE on excitation length of pump beam for different pump intensities. (a) $51 \mathrm{~kW} \mathrm{~cm}^{-2}$; (b) $158 \mathrm{~kW} \mathrm{~cm}^{-2}$; (c) $240 \mathrm{~kW} \mathrm{~cm}^{-2}$ and (d) $793 \mathrm{~kW} \mathrm{~cm}^{-2}$.

from the plot, corresponding to the change of slope, a threshold pump intensity of around $51 \mathrm{~kW} \mathrm{~cm}{ }^{-2}$ was observed for the occurrence of ASE.

On a detailed study of the emission spectrum of the dye doped thin film, it was found that the emission spectrum exhibits all the features of ASE emission, namely the property of directionality, narrow linewidth and presence of soft threshold behaviour [25]. Directionality of the output beam was so obvious that no focusing was required to collect the beam. The linewidth was reduced to $3.5 \mathrm{~nm}$ for a pump intensity of $825 \mathrm{~kW} \mathrm{~cm}^{-2}$. A threshold pump intensity of $51 \mathrm{~kW} \mathrm{~cm}^{-2}$ was observed for the occurrence of ASE.

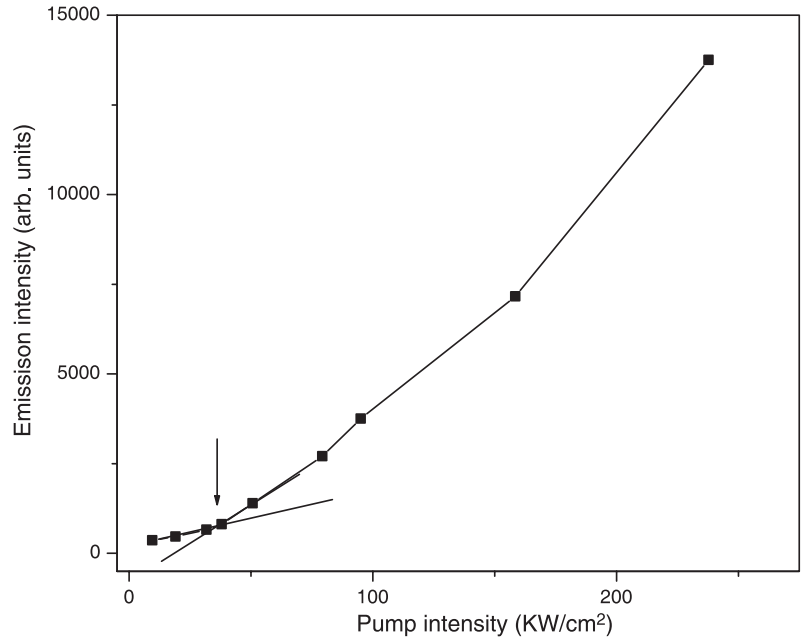

Figure 4. Variation of emission intensity with pump intensity for an excitation length of $2 \mathrm{~mm}$.

\subsection{Laser emission}

In most of the earlier reports of ASE exhibited by dye doped polymer films, gain saturation was observed at longer excitation lengths of the gain medium [13-18]. Once gain saturation occurs, light is not amplified further as the length of the stripe is increased. At gain saturation, line narrowing ceases and the FWHM of the emission is found to be a constant which is usually less than $10 \mathrm{~nm}$. In contrast to this, in the present study, when the stripe length was increased beyond 2$3 \mathrm{~mm}$, a sudden line narrowing was observed in the emission spectrum at all pump intensities above the threshold for ASE 


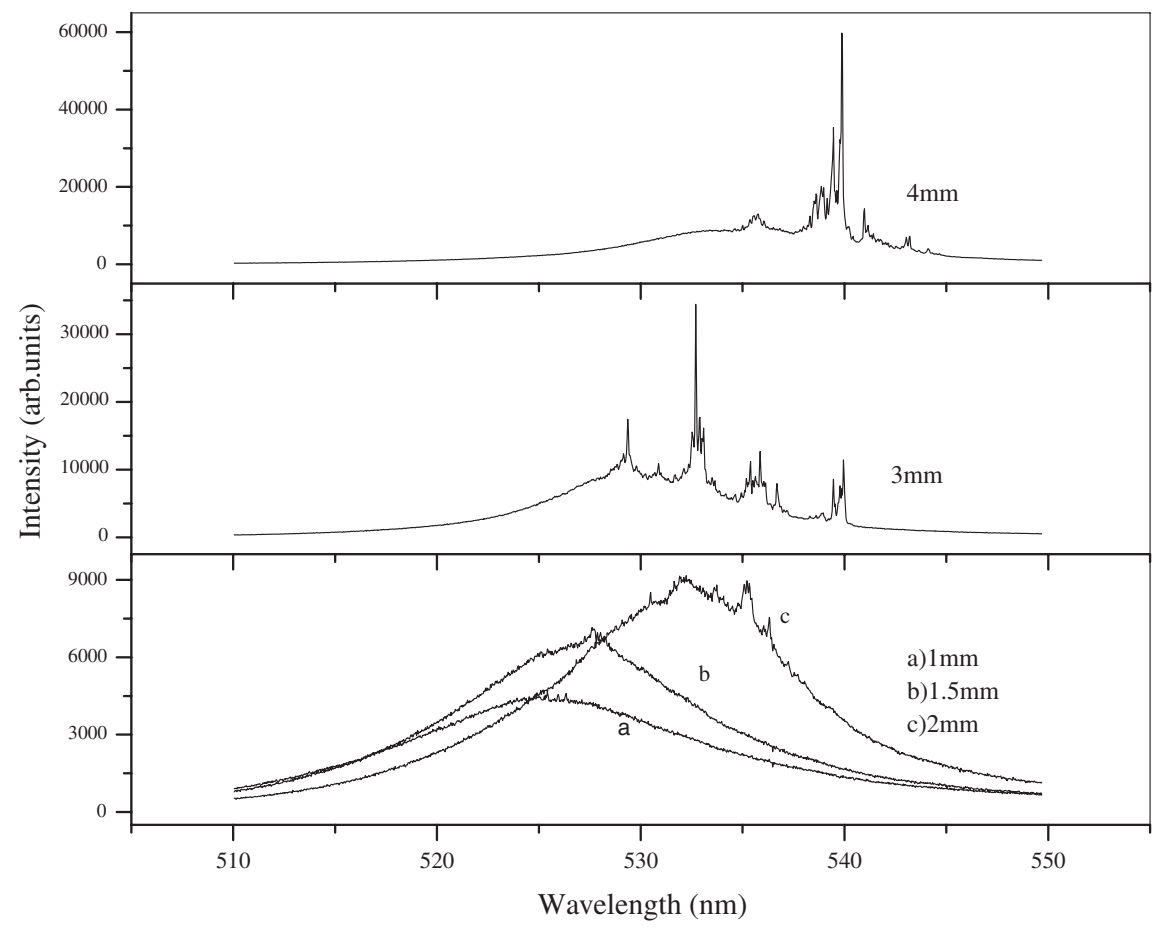

Figure 5. Plot of ASE and laser emission from a dye doped film with the increase in excitation length. Pump intensity is $79 \mathrm{~kW} \mathrm{~cm}^{-2}$.

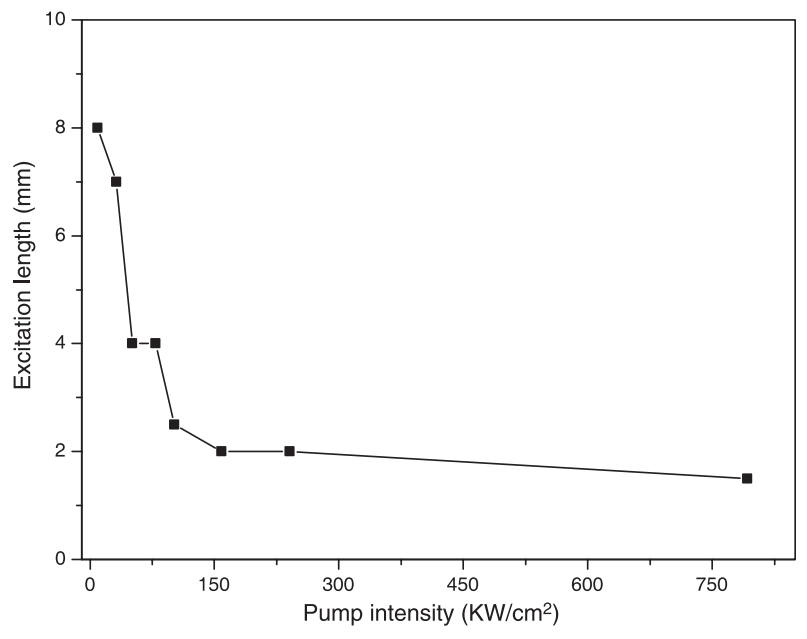

Figure 6. Plot of excitation length versus threshold pump intensity for laser emission.

(figure 5). The FWHM of the spectral lines was less than $1 \mathrm{~nm}$. Such a spectral narrowing can be attributed to laser emission. The spectral narrowing was very prominent at higher pump intensities where the FWHM was reduced to a value of $0.3 \mathrm{~nm}$. The output intensity was also found to increase with pump intensity but not in an exponential manner. For different pump intensities, the excitation length of the medium needed for laser emission was found to be different. For a pump intensity of $51 \mathrm{~kW} \mathrm{~cm}^{-2}$, an excitation length of $3 \mathrm{~mm}$ gave rise to laser emission but it was reduced to $1.75 \mathrm{~mm}$ for a pump intensity of $825 \mathrm{~kW} \mathrm{~cm}^{-2}$. Figure 6 denotes the variation of excitation length with the threshold pump intensity that produced a laser emission.
Laser emission requires an external feedback. In the present case no feedback is provided with external mirrors. But the free-standing film with air medium on both sides can be considered as an optical cavity. The lateral faces of the films are of good optical quality and they behave like external mirrors partially reflecting the beam, though the reflectivities are very small compared to conventional mirrors. These reflecting surfaces produce the effect of a Fabry-Perot optical cavity whose length is the thickness of the film.

The excited active medium can be considered as a number of serially connected Fabry-Perot optical cavities [24]. The axis of the optical cavity is normal to the direction of propagation of light through the film. The multiple pass between the film surfaces directly increases the gain. When the gain of the active medium compensates the losses in the medium, laser emission occurs. According to laser theory the gain per pass in the active medium is $\exp \sigma\left(N_{2}-\left(g_{2} / g_{1}\right) N_{1}\right) l$, where $\sigma$ is the stimulated emission cross section and $l$ is the length of the active medium [25].

Due to the sample geometry and mode of excitation, there can be a guiding effect in the gain medium even though the film thickness is around 100-200 $\mu \mathrm{m}$. The excited portion of the thin film behaves like a planar waveguide. The axial modes of the F-P cavity can be considered as the transverse modes of the waveguide [24]. When the excitation length of the gain medium and the pump intensity are sufficiently increased, laser emission is observed. Thus both the guiding effect in the direction of the excited stripe and the feedback from the lateral faces of the thin film induce the high gain necessary for the laser emission. Since the active medium is highly optically dense, even a small length of $1.75 \mathrm{~mm}$ of the medium could produce laser emission with a pump intensity of $825 \mathrm{~kW} \mathrm{~cm}^{-2}$. 


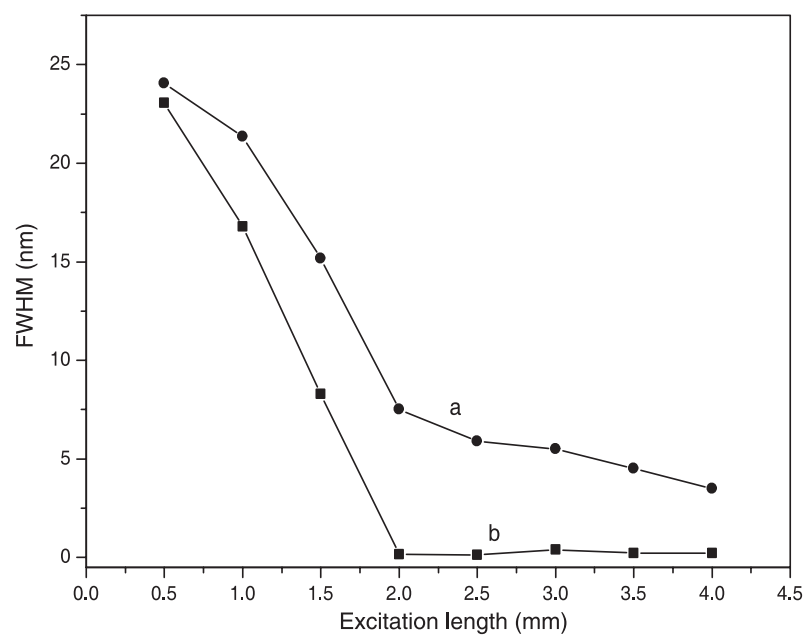

Figure 7. Dependence of linewidth on excitation length of pump beam for (a) ASE for pump intensity of $79 \mathrm{~kW} \mathrm{~cm}^{-2}$ and (b) laser emission for a pump intensity of $240 \mathrm{~kW} \mathrm{~cm}^{-2}$.

In the line narrowing regime, the FWHM of the emission line was below $1 \mathrm{~nm}$ which further did not show any significant change with excitation length. Figure 7 clearly gives a comparison of reduction in FWHM for ASE and laser emission. The values of the excitation stripe length needed for spectral narrowing, wavelength of peak emission, the magnitude of the line narrowing and the red shift observed for different excitation lengths were sensitive to certain physical and chemical characteristics of the sample. These variations may be attributed to the differences in microscopic structure of the polymer thin films which occurs during the fabrication process.

\subsection{Laser resonant modes}

The existence of a Fabry-Perot optical cavity between the lateral faces of the free-standing film was confirmed by the occurrence of well-resolved discrete peaks in the emission spectrum. A number of equally spaced resonant modes were observed (figure 8). These modes can be considered as the axial modes of the Fabry-Perot cavity formed between the flat film surfaces [24]. The spacing of the modes was found to be around $1.1 \mathrm{~nm}$. In general the spacing between the modes is given by $\Delta \lambda=\lambda^{2} / 2 n L$, where $n$ is the refractive index of the medium and $L$ is the optical path length of the resonator cavity. By substituting $n$ as 1.49 , the refractive index of undoped PMMA, $\lambda$ the peak value observed in the spectrum, and $L$, the thickness of the polymer film in the above equation, the spacing was calculated to be $1.02 \mathrm{~nm}$ for a $90 \mu \mathrm{m}$ thick film which was in agreement with the observed value of $\sim 1.1 \mathrm{~nm}$. To confirm this observation, dye doped films with different thicknesses were studied. With the film of thickness $190 \mu \mathrm{m}$, the spacing observed was $\sim 0.5 \mathrm{~nm}$ against the theoretical value of $0.55 \mathrm{~nm}$.

\section{Conclusion}

We report the occurrence of both amplified spontaneous emission and laser emission from Coumarin 540 dye doped

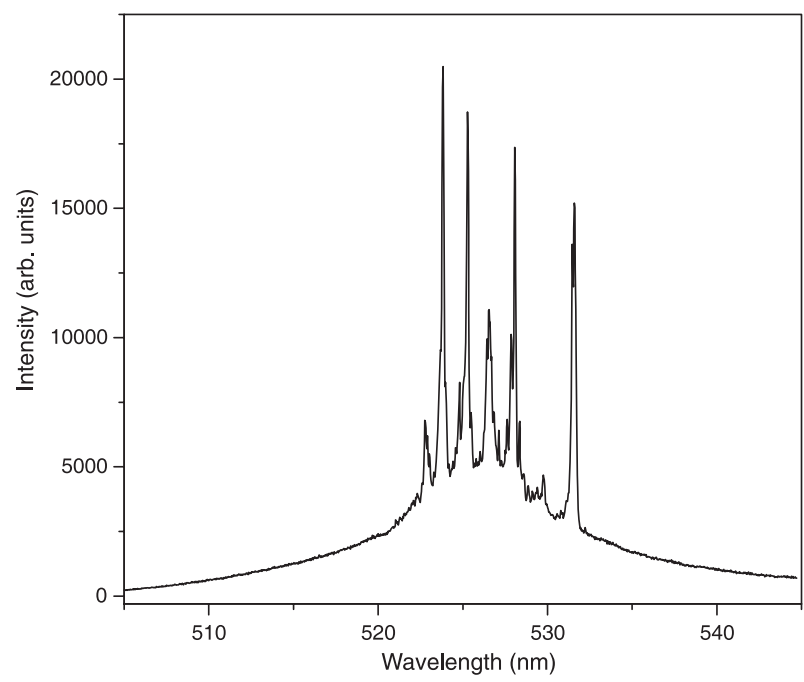

Figure 8. Resonant modes with a spacing of $1.1 \mathrm{~nm}$ from a $90 \mu \mathrm{m}$ thick dye doped film. Pump intensity $51 \mathrm{~kW} \mathrm{~cm}^{-2}$. Excitation length is $4 \mathrm{~mm}$.

PMMA polymer films. The phenomena are highly dependent on the excitation length of the medium. ASE is observed when the excitation length of the active medium is below $2-3 \mathrm{~mm}$. An increase in excitation length shows a shift from ASE to laser emission for a threshold pump intensity of $51 \mathrm{~kW} \mathrm{~cm}^{-2}$. With a pump intensity of $825 \mathrm{~kW} \mathrm{~cm}^{-2}$, the dye doped films give rise to laser emission at an excitation length as small as $1.75 \mathrm{~mm}$. Coumarin 540 doped polymer thin film is found to be a highly efficient gain medium which can produce laser emission at such a short length. Optical feedback from the lateral faces of the thin film and the guiding effect due to the sample geometry and mode of excitation have contributed to the gain needed for laser emission. Well-resolved laser modes with equal spacing are also observed from the dye doped films which confirm the optical feedback from the Fabry-Perot-like optical cavity formed between the polished surfaces of the thin film. These results establish the occurrence of lasing action in dye doped polymer films having micrometer thickness and could be of considerable application in the design of active optical IC chips.

\section{Acknowledgments}

The first author is grateful to the University Grants Commission, New Delhi, India for the financial support in the form of a FIP grant. Assistance from UGC under the UPE program also is gratefully acknowledged.

\section{References}

[1] Costela A, Garcia-Morena I and Figuera J M 1998 Laser Chem. 18 63-84

[2] Sastre R and Costela A 1995 Adv. Mater. 7 198-202

[3] Lo D, Parris J E and Lawless J L 1992 Appl. Phys. B 55 365-7

[4] Peterson O G and Snavely B B 1968 Appl. Phys. Lett. 12 238-40

[5] Diaz-Garcia M A, Fernandez S and Kuzyk M G 2002 Appl. Phys. Lett. 80 4486-8 
[6] Alexander I 2002 Polym. Adv. Technol. 13 744-52

[7] Sorek Y, Reisfeld R, Finkelstein I and Rushin S 1995 Appl. Phys. Lett. 66 1169-71

[8] Duarte F J and James R O 2003 Opt. Lett. 28 2088-91

[9] Gaur S S, Ghawana K, Sharma V K and Tripathi K N 2004 J. Opt. A: Pure Appl. Opt. 6 312-4

[10] Jiu Y L, Laquai F, Ziegler A and Wegner G 2005 Proc. 8th Polymers for Advanced Technologies Int. Symp. pp 1-3

[11] Duarte F J, Liao L S, Vaeth K M and Miller A M 2006 J. Opt. A: Pure Appl. Opt. 8 172-4

[12] McGehee M D, Gupta R, Veenstra S, Miller E K, Diaz-Garcia M A and Heeger A J 1998 Phys. Rev. B 58 7035-9

[13] Lu W, Zhong B and Ma D 2004 Appl. Opt. 43 5074-8

[14] Brouwer H J, Krasnikov V V, Hilberer A and Hadziioanno G 1997 Adv. Mater. 8 61-75

[15] Deshpande A V and Namdas E B 1997 Appl. Phys. B 64 419-22

[16] Otomo A, Yokoyama S, Nakahama T and Mashiko S 2000 Appl. Phys. Lett. 77 3881-3
[17] Kretsch K P, Belton C, Lipson S, Blau W J, Henari F Z, Rost H, Pfeiffer S, Teuschel A, Tillmann $\mathrm{H}$ and Horhold H 1999 J. Appl. Phys. 86 6155-8

[18] Berg S A, Schoonderwoerd R H, Schoo H F M, Hooft G W and Eliel E R 1999 Opt. Lett. 24 1847-9

[19] Walter K 1972 Solid State Laser Engineering (Berlin: Springer) pp 182-5

[20] Yokoyama S, Otomo A and Mashiko S 2002 Appl. Phys. Lett. 80 7-9

[21] Gruner J, Cacialli F and Friend R H 1996 J. Appl. Phys. 80 207-15

[22] Helbo B, Kristensen A and Menon A 2003 J. Micromech. Microeng. 13 307-11

[23] Sobel F, Gindre D, Nunzi J M, Denis C, Dumarcher V, Debuisschert C F, Kretsch K P and Rocha L 2004 Opt. Mater. 27 199-201

[24] Geetha K, Rajesh M, Nampoori V P N, Vallabhan C P G and Radhakrishnan P 2006 J. Opt. A: Pure Appl. Opt. 8 189-93

[25] Svelto O and Hanna D C 1998 Principles of Lasers 4th edn (New York: Plenum) pp 71-3 